\title{
Use of a colorimetric (DELI) test for the evaluation of chemoresistance of Plasmodium falciparum and Plasmodium vivax to commonly used anti-plasmodial drugs in the Brazilian Amazon
}

Lilian R Pratt-Riccio ${ }^{1,2}$, Yonne F Chehuan ${ }^{3}$, Maria José Siqueira ${ }^{3}$, Maria das Graças Alecrim ${ }^{3}$, Cesare Bianco-Junior ${ }^{1,2}$, Pierre Druilhe ${ }^{4}$, Philippe Brasseur ${ }^{5}$, Maria de Fátima Ferreira-da-Cruz ${ }^{1,2}$, Leonardo JM Carvalho ${ }^{1,2}$ and Cláudio T Daniel-Ribeiro ${ }^{1,2^{*}}$

\begin{abstract}
Background: The emergence and spread of Plasmodium falciparum and Plasmodium vivax resistance to available anti-malarial drugs represents a major drawback in the control of malaria and its associated morbidity and mortality. The aim of this study was to evaluate the chemoresistance profile of $P$. falciparum and $P$. vivax to commonly used anti-plasmodial drugs in a malaria-endemic area in the Brazilian Amazon.

Methods: The study was carried out in Manaus (Amazonas state), in the Brazilian Amazon. A total of 88 P. falciparum and 178 P. vivax isolates was collected from 2004 to 2007. The sensitivity of $P$. falciparum isolates was determined to chloroquine, quinine, mefloquine and artesunate and the sensitivity of $P$. vivax isolates was determined to chloroquine and mefloquine, by using the colorimetric DELI test.
\end{abstract}

Results: As expected, a high prevalence of $P$. falciparum isolates resistant to chloroquine (78.1\%) was observed. The prevalence of isolates with profile of resistance or decreased sensitivity for quinine, mefloquine and artesunate was 12.7, 21.2 and $11.7 \%$, respectively. In the case of $P$. vivax, the prevalence of isolates with profile of resistance for chloroquine and mefloquine was 9.8 and $28 \%$, respectively. No differences in the frequencies of isolates with profile of resistance or geometric mean IC50s were seen when comparing the data obtained in 2004, 2005, 2006 and 2007, for all tested anti-malarials.

Conclusions: The great majority of $P$. falciparum isolates in the Brazilian malaria-endemic area remain resistant to chloroquine, and the decreased sensitivity to quinine, mefloquine and artesunate observed in 10-20\% of the isolates must be taken with concern, especially for artesunate. Plasmodium vivax isolates also showed a significant proportion of isolates with decreased sensitivity to chloroquine (first-line drug) and mainly to mefloquine. The data presented here also confirm the usefulness of the DELI test to generate results able to impact on public health policies.

Keywords: Malaria, Plasmodium falciparum, Plasmodium vivax, Resistance, Anti-malarials, DELI test

\footnotetext{
* Correspondence: ribeiro@ioc.fiocruz.br

'Laboratório de Pesquisas em Malária, Instituto Oswaldo Cruz, Fiocruz,

Avenida Brasil 4365, Manguinhos, Rio de Janeiro, RJ, Brazil CEP: 21040-900

${ }^{2}$ Centro de Pesquisa, Diagnóstico e Treinamento em Malária (CPD-Mal),

Fiocruz, Reference Center for Malaria in the Extra-Amazonian Region for the

Secretary for Health Surveillance from the Ministry of Health, Rio de Janeiro,

RJ, Brazil

Full list of author information is available at the end of the article
} 


\section{Background}

Malaria is one of the most serious public health problems worldwide. It is a leading cause of disease and death in many developing countries, where young children and pregnant women are the most affected groups. Malaria is endemic in 99 countries and, according to the World Health Organization (WHO), there were about 219 million cases of malaria and an estimated 660,000 malaria deaths in 2010 [1].

In Brazil, malaria is hypo- to meso-endemic, present throughout the year with clear seasonal fluctuations and is frequently associated with migration movements of non-immune individuals to areas where malaria is endemic [2]. In 2011, approximately 263,000 malaria cases were reported in Brazil, $99.8 \%$ of them in the Amazon Region [3].

In the absence of an effective vaccine, malaria control is almost entirely dependent on the availability of effective chemotherapy and the efficacy of anti-vectorial measures. The emergence and spread of Plasmodium falciparum resistance to anti-malarial drugs thus represent a major drawback in the control of malaria and its associated morbidity and mortality. Resistance to current available anti-malarial drugs has been reported in many places in the world, including Brazil. The case of chloroquine is representative. Chloroquine appeared in the 1940s as an extraordinary anti-malarial drug: effective, cheap and easy to produce. However, since the first reports of $P$. falciparum parasites with reduced sensitivity to chloroquine [4-7], the resistance to this drug spread rapidly throughout the world. In many places, it became no longer effective and had to be substituted by other drugs, more expensive and in many cases presenting considerable side effects. The same was true in Brazil, where the first report of $P$. falciparum resistance to chloroquine was in the 1960s [4] and, by 1990, more than $95 \%$ of $P$. falciparum isolates circulating in malaria endemic areas were resistant to chloroquine [8].

Given the strong impact of chemoresistance to antimalarial drugs in the control of malaria, monitoring the development of resistant phenotypes is a priority wherever malaria is endemic. The in vitro methods for evaluating anti-malarial drug sensitivity can provide a profile of plasmodial sensitivity to a variety of drugs, since several drugs can be assayed simultaneously. A lower sensitivity in vitro of an isolate does not necessarily mean that it will be resistant in vivo, because the in vivo outcome depends on a number of factors that cannot be evaluated in vitro. However, it can provide an outline of resistant phenotypes circulating for each drug tested when using samples from an adequate number of patients in a given area, thus providing crucial information for policymakers to determine the best drug to be employed in the area. Ideally, monitoring of antimalarial chemoresistance must be continuous, since development and spread of resistance are dynamic events, changing with time and according to human interventions and other factors, such as population migration.

A good alternative for microscopic and isotopic in vitro methods appeared with the development of immunoenzymatic methods to evaluate plasmodial growth. The double-site enzyme-linked immunodetection (DELI) test $[9,10]$ is based on the detection of the parasite lactate dehydrogenase (LDH), produced during the plasmodial growth, by means of monoclonal antibodies, in a sandwich ELISA. Deli-test assay proved to be reproducible, very sensitive to detect low parasitaemia $(0.005 \%)$ and easy to perform even in field conditions, and without the need of well training microscopists or manipulation of [3] hypoxanthine [10-13]. As an ELISA procedure, it allows the fast evaluation of many samples at once. In addition, DELI-test is easier to perform, faster to implement, and cheaper than in vitro isotopic assays [10]. With the development of an efficient short-term culture protocol for in vitro Plasmodium vivax culture [14], and the availability of monoclonal antibodies specific for P.vivax LDH, DELI test also allows the study of the sensitivity of this parasite to anti-malarial drugs.

The aim of this study was to evaluate the chemoresistance profile of $P$. falciparum and $P$. vivax to commonly used anti-plasmodial drugs in a Brazilian malaria-endemic area in the Amazon Region using the DELI-test.

\section{Methods}

\section{Studied area and isolates}

This study was carried out in the city of Manaus (Amazonas state), located at the Brazilian Amazon. A total of $88 P$. falciparum and $178 \mathrm{P}$. vivax isolates were collected from patients who sought health care at Dr Heitor Vieira Dourado Tropical Medicine Foundation (FMT-HVD) from 2004 to 2007.

Written informed consent was obtained from all donors and venous blood samples were drawn in Vacutainer ${ }^{\odot}$ (Becton Dickinson, Oxnard, CA) EDTA tubes. Thin and thick blood smears were examined for identification of malaria parasite and determination of parasitaemia by two expert malaria microscopists from FMT-HVD and from the Laboratory of Malaria Research (Fiocruz, Rio de Janeiro, Brazil) which is a reference centre in malaria diagnosis for the Brazilian Ministry of Health. Thick blood smears from all subjects were stained with Giemsa and examined under 1,000-fold magnification. Parasitaemia was determined by counting parasites in reference to 200 white blood cells in thick blood films, and the number of the blood parasites per millilitre was calculated.

All malaria patients enrolled in this study complied with the following criteria: 1 ) they presented symptoms; 2) they had $P$. falciparum or $P$. vivax infection; 3 ) they neither used chemoprophylaxis nor took anti-malarial 
drugs as self-treatment; 4 ) they were 12 years old or older; 5) females were not pregnant or breast feeding; and, 6) blood collection was performed at the day of diagnosis before malaria treatment. After malaria diagnosis and blood sample collection, the patients were immediately treated according to the Brazilian Ministry of Health standards for malaria therapy.

The study was reviewed and approved by the Fundação Oswaldo Cruz Ethical Committee (number 221/03).

\section{Drug sensitivity assay}

The sensitivity of $P$. falciparum isolates was determined to chloroquine sulphate, quinine hydrochloride, mefloquine hydrochloride, and artesunate and the sensitivity of $P$. vivax isolates was determined to chloroquine sulphate and mefloquine hydrochloride (all drugs obtained from Sigma-Aldrich).

Anti-malarial drugs aliquoted in predosed tubes ( $15 \mathrm{mg} /$ tube) were dissolved by the addition of $3 \mathrm{~mL}$ of $100 \%$ ethanol and $7 \mathrm{~mL}$ of RPMI-1640 medium (Gibco, Invitrogen Life Technologies) (chloroquine and quinine) or $10 \mathrm{~mL}$ of $100 \%$ methanol (mefloquine and artesunate). From the stock solution, another solution was prepared for each drug at final concentrations of $2,400 \mathrm{ng} / \mathrm{mL}$ for chloroquine, $1,200 \mathrm{ng} / \mathrm{mL}$ for quinine and $150 \mathrm{ng} / \mathrm{mL}$ for mefloquine and artesunate in RPMI-1640 medium supplemented with $0.5 \%$ albumax (Gibco) (complete culture medium), for P. falciparum; and $600 \mathrm{ng} / \mathrm{mL}$ for chloroquine and $300 \mathrm{ng} / \mathrm{mL}$ for mefloquine in a 3:1 mix (vol/vol) of RPMI 1640 medium and Waymouth medium (Sigma-Aldrich), for $P$. vivax. One hundred $\mathrm{mL}$ of each dilution were added to wells of the column 1 of 96-well tissue culture plates (Falcon) and nine subsequent two-fold dilutions were prepared in wells 2-9. Wells 10-12 were filled with $50 \mu \mathrm{L}$ of complete culture medium (culture control wells). Each concentration of anti-malarial was tested in duplicate ( $P$. falciparum) or quadruplicate ( $P$. vivax).

Isolates with at least $0.1 \%$ parasitaemia were included in the study and were maintained at $4^{\circ} \mathrm{C}$ (for up to $8 \mathrm{hr}$ after collection) before the in vitro culture was started. Blood samples were washed twice with a solution of RPMI-1640 medium and then resuspended in the complete culture medium (P. falciparum) or RPMIWaymouth $(P$. vivax). If necessary, a dilution was performed by adding uninfected O-positive-group erythrocytes to obtain a $0.5-1 \%$ parasite density. Finally, $200 \mu \mathrm{L}$ of this suspension were distributed to each well in the anti- malarial predosed plates in $1.2 \%$ final haematocrit. The plates were incubated for $48 \mathrm{hr}$ at $37^{\circ} \mathrm{C}$ in a $\mathrm{CO}_{2}$ incubator $\left(5 \% \mathrm{CO}_{2}\right.$ in air) and were then frozen and kept at $-20^{\circ} \mathrm{C}$. Before ELISA, the plates were frozen and thawed three times to lyse the red blood cells.

\section{ELISA}

The success of the drug sensitivity assay and the appropriate volume of haemolysed culture were previously determined for each clinical isolate by using a preliminary LDH ELISA as a pretest. To determine which dilution of haemolysed culture had to be used in the DELI-test, four serial dilutions $(1: 50,1: 25,1: 12.5,1: 6.25)$ of culture control wells (no drug) of each isolate were tested in a preliminary LDH ELISA. The dilutions used were those providing optical density (OD) readings between 1.0-2.0.

ELISA plate (Nunc, Maxisorb, Denmark) wells were coated with $100 \mu \mathrm{L}$ of monoclonal antibody (MAb) against P. falciparum (17EA) or P. vivax (11D) LDH (kind gifts of Dr Michael Makler, Flow Inc, Portland, USA) at 1 $\mu \mathrm{g} / \mathrm{mL}$, in PBS $\mathrm{pH}$ 7.4. The plates were incubated overnight at $4{ }^{\circ} \mathrm{C}$, washed with PBS with $1 \%$ BSA (fraction V, Boehringer-Mannheim) (PBS-BSA) and then incubated with $300 \mu \mathrm{L}$ of PBS-BSA for $4 \mathrm{hr}$, at room temperature. The plates were maintained at $4^{\circ} \mathrm{C}$ until used.

Subsequently, the appropriated volume of the haemolysed culture was transferred to the ELISA plate wells with PBS-BSA to the final volume of $100 \mu \mathrm{L}$, incubated for $1 \mathrm{hr}$ at $37^{\circ} \mathrm{C}$ and then washed with PBS-BSA. After the addition of $100 \mu \mathrm{L}$ per well of a biotinylated MAb against pan-Plasmodium LDH (19G7), the plates were incubated for $1 \mathrm{hr}$ at $37^{\circ} \mathrm{C}$. After washing, a third incubation for $30 \mathrm{~min}$ at room temperature with $100 \mu \mathrm{L}$ of a streptavidin horseradish peroxidase solution was followed by a last washing step. Enzyme activity was revealed by incubation for $5 \mathrm{~min}$ at room temperature with $100 \mu \mathrm{L}$ of tetramethylbenzidine (TMB). The reaction was stopped with $1 \mathrm{M}$ of phosphoric acid, and the absorbance was read at $450 \mathrm{~nm}$ in a spectrophotometer (Spectramax 250, Molecular Devices).

Concentration-response data were analysed by a nonlinear regression function to determine the $50 \%$ inhibition of parasite growth (IC50), defined as the concentration of the drug which inhibited $50 \%$ of the production of $\mathrm{LDH}$ as determined by OD values from sample test wells compared to those obtained from drug-free control wells. The IC50 threshold values for resistance to chloroquine,

Table 1 Frequencies of Plasmodium falciparum and Plasmodium vivax field isolates from Manaus, Amazonas State, Brazil, with IC50 of commonly used anti-malarial drugs determined in 2004, 2005, 2006 and 2007

\begin{tabular}{llllll}
\hline & $\mathbf{2 0 0 4} \mathbf{~ n d} / \mathbf{n}(\%)$ & $\mathbf{2 0 0 5} \mathbf{~ n d} / \mathbf{n}(\%)$ & $\mathbf{2 0 0 6} \mathbf{~ n d} / \mathbf{n}(\%)$ & $\mathbf{2 0 0 7} \mathbf{~ n d} / \mathbf{n}(\%)$ & Total $\mathbf{n d} / \mathbf{n}(\%)$ \\
\hline P. falciparum & $16 / 30(53)$ & $18 / 19(94)$ & $20 / 22(91)$ & $16 / 17(94)$ & $70 / 88(80)$ \\
$P$. vivax & $11 / 23(48)$ & $24 / 44(55)$ & $32 / 38(84)$ & $65 / 73(89)$ & $132 / 178(74)$ \\
\hline
\end{tabular}

nd: number of samples with IC50 determined; $n$ : number of total samples (percentage). 
mefloquine, quinine and artesunate were $100 \mathrm{nM}, 30 \mathrm{nM}$, $500 \mathrm{nM}$ and $10 \mathrm{nM}$, respectively; these values were similar to those already described elsewhere $[10,11,15,16]$.

\section{Statistical analysis}

The data were stored in the Fox-plus (Borland International Inc, Perrysburg, OH, USA) data bank software. GraphPad Instat and GraphPad Prism (GraphPad Software Inc, CA, USA) statistical software programs were used for data analysis. The Student's t-test was used to analyse the differences in IC50 mean values and the Chi-square test was applied to compare the prevalence of isolates with profile of resistance observed from 2004 to 2007.

\section{Results}

A total of 88 P. falciparum and 178 P. vivax samples was collected from malaria patients from 2004 to 2007. Males were predominant in both $P$. falciparum $(71 \%)$ and $P$. vivax (81\%) groups. The average age was also similar between $P$. falciparum ( $36 \pm 15$ years old) and $P$. vivax (34 \pm 13 years old) groups.

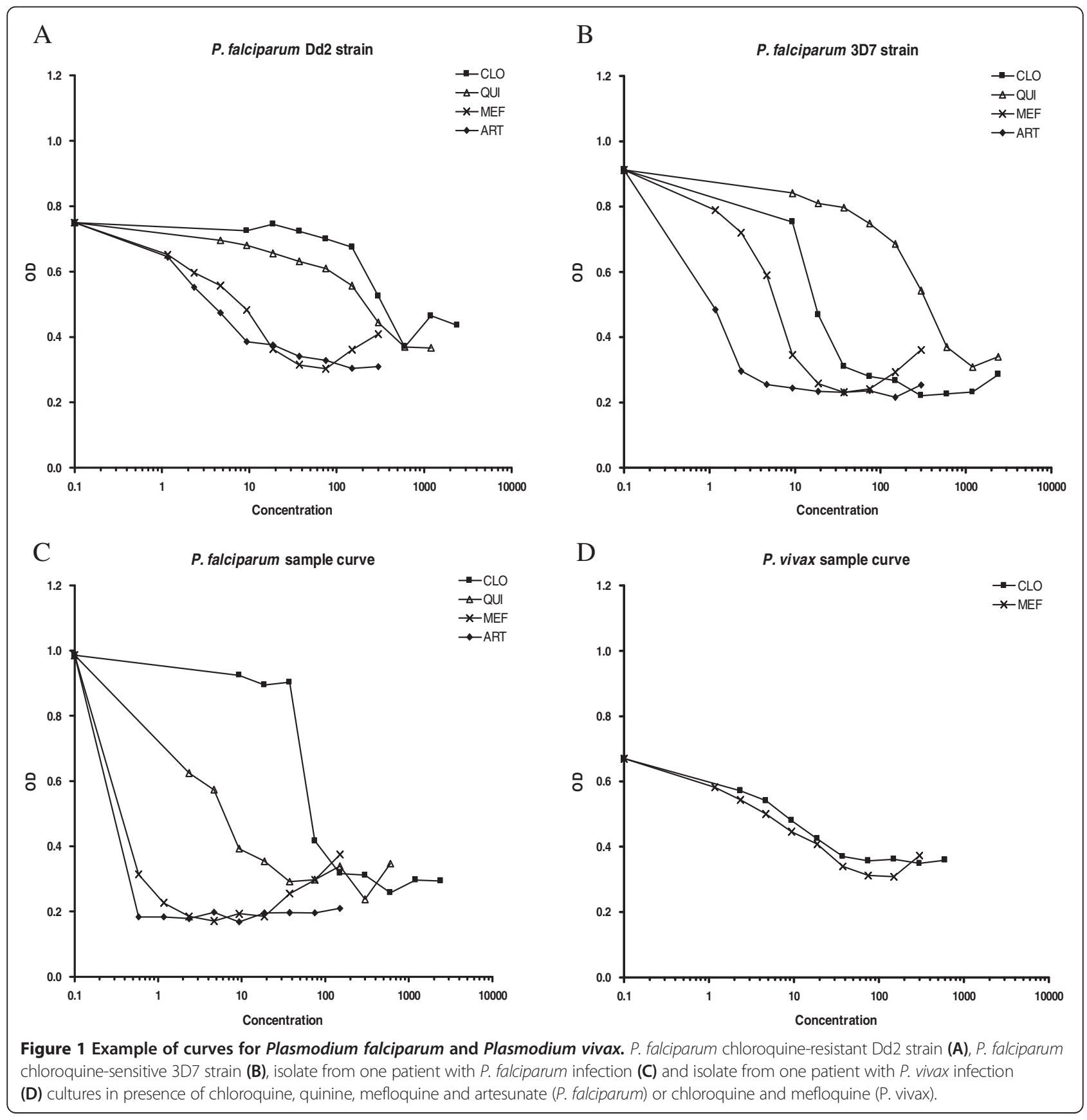



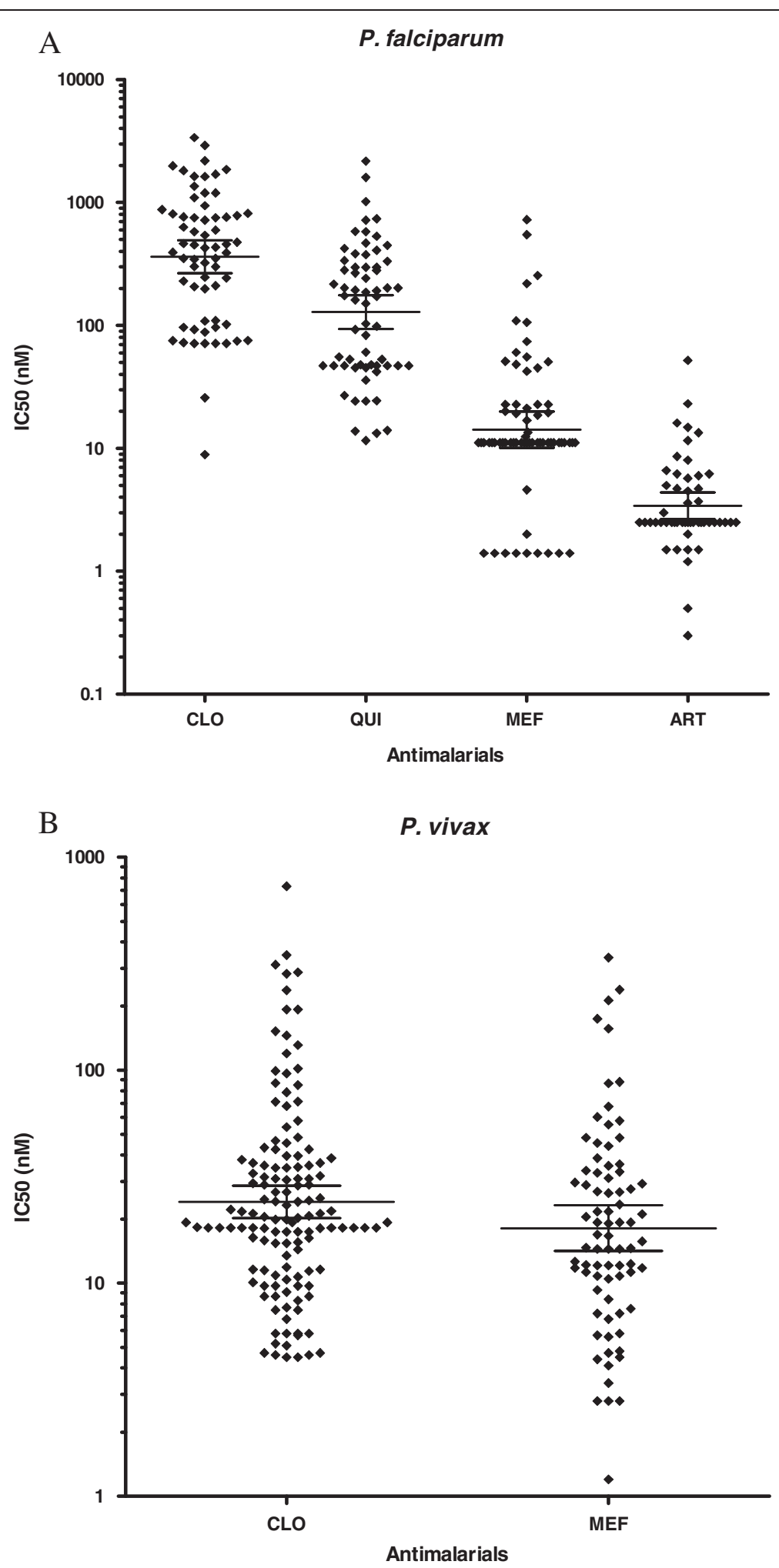

Figure 2 Distribution of IC50 values in Plasmodium falciparum and Plasmodium vivax isolates using DELI test. Plasmodium falciparum isolates (A), Plasmodium vivax isolates (B). Values are individual IC50 (nM). Lines represent geometric mean with 95\% confidence interval. 
Overall, the IC50 values could be determined in most of P. falciparum (70/88, 80\%) and P. vivax (132/178, 74\%) samples (Table 1). Successful in vitro culture allowing IC50 determination for fresh isolates was low in the first year (2004) for both $P$. falciparum and $P$. vivax cultures, but substantially increased in the subsequent years, suggesting better handling and optimization of culture procedures (Table 1). The feasibility of the procedure using chloroquine-sensitive (Dd2) and chloroquine-resistant (3D7) strains adapted for in vitro culture was determined and showed that it worked well (Figure 1).

As expected, a high prevalence of $P$. falciparum isolates resistant or with decreased susceptibility to chloroquine was observed (Figure 2A). Indeed, 78.1\% (50/64) of the samples showed IC50 higher than $100 \mathrm{nM}$. The geometric mean IC50s for chloroquine was $361.8 \mathrm{nM}$ (95\% confidence interval 266-492 nM) (Table 2). For quinine, the prevalence of clearly resistant isolates (IC50 > 500 nM) was 12.7\% $(8 / 63)$. The geometric mean IC50s for quinine was 128.5 nM (95\% confidence interval 93-175). For mefloquine, the prevalence of isolates with profile of resistance or decreased susceptibility (IC50 > 30nM) was 21.2\% (14/66). The geometric mean IC50s for mefloquine was $14.2 \mathrm{nM}$ (95\% confidence interval 10-20). Finally, for artesunate, the prevalence of isolates with IC50 above the threshold of $10 \mathrm{nM}$ was $11.7 \%(6 / 51)$. The geometric mean of IC50s was $3.4 \mathrm{nM}$ (95\% confidence interval 3-4). No difference in frequencies of isolates with profile of resistance or geometric mean IC50s were seen comparing the data obtained in 2004, 2005, 2006 and 2007 for chloroquine, quinine, mefloquine and artesunate.

Among P. falciparum isolates, different chloroquine, quinine, mefloquine, and artesunate sensitivity levels were observed, however, the temporal analysis indicate that the profile of sensitivity to these drugs remained relatively stable.

In the case of $P$. vivax isolates, the prevalence of isolates with IC50 above threshold of $100 \mathrm{nM}$ for chloroquine was 9.8\% (13/132) (Figure 2B) and the geometric mean IC50 was $24.1 \mathrm{nM}$ (95\% confidence interval 20-29). For mefloquine, the prevalence of IC50 above threshold of $30 \mathrm{nM}$ was $28 \%(22 / 78)$ and the geometric mean IC50 was $18.1 \mathrm{nM}$ (95\% confidence interval 14-23) (Table 2). No difference in frequencies of isolates with profile of resistance or geometric mean IC50s were seen comparing the data obtained in 2004, 2005, 2006 and 2007 for chloroquine and mefloquine.

\section{Discussion}

In the present study, the DELI-test was used to determine the profile of in vitro sensitivity of $P$. falciparum and $P$. vivax field isolates in Manaus, in the Brazilian Amazon. Despite some initial technical difficulties in the beginning of the study (2004), the performance of the test in 2005-2007 was similar to that observed in previous studies in Africa, which reported test success rates of $81 \%$ for P. falciparum [13].

Overall, a high prevalence (78.1\%) of P. falciparum isolates resistant or with decreased sensitivity to chloroquine was observed. This was expected since earlier studies conducted in Amazonia suggested that $100 \%$ of the $P$. falciparum isolates circulating in the area were chloroquineresistant [17]. In addition, most of the "sensitive" isolates had IC50s close to the threshold for sensitivity $(100 \mathrm{nM})$ and therefore represent rather borderline isolates. Only 2/64 (3.1\%) of isolates showed clearly low IC50 to chloroquine. Any evidence of the re-emergence of chloroquine-sensitive parasites in Brazil through the documentation of a wild pfcrt P. falciparum haplotype (CVMNK) in Amazonian region was already reported [18].

In Brazil, reduced in vitro and in vivo sensitivity to mefloquine has been reported since 1981 [19-23] and this might explain the $21.2 \%$ resistant phenotypes in the present study. In fact, even before the official introduction of mefloquine in Brazilian endemic areas in 1987, P. falciparum isolates with profile of resistance to mefloquine had already been reported [24].

In relation to quinine, the prevalence of resistant isolates (IC50 above $500 \mathrm{nM}$ ) was $12.7 \%$. Some isolates showed IC50s very close to the threshold. In any case, this scenario is worrisome because a continuous decreasing in parasite sensitivity has been noted in the Brazilian Amazon $[17,21,24-27]$ and quinine plus doxycycline is actually

Table 2 IC50 values of commonly used anti-malarial drugs in in vitro culture of Plasmodium falciparum and in Plasmodium vivax from Manaus, Amazonas State, Brazil

\begin{tabular}{|c|c|c|c|c|c|c|c|c|}
\hline \multirow[b]{3}{*}{ Drug $^{1}$} & \multicolumn{4}{|l|}{ P. falciparum } & \multicolumn{4}{|l|}{ P. vivax } \\
\hline & \multirow[b]{2}{*}{ No. of isolates } & \multicolumn{3}{|l|}{ IC50 (nM) } & \multirow[b]{2}{*}{ No of isolates } & \multicolumn{3}{|l|}{ IC50 (nM) } \\
\hline & & Geometric mean & $95 \% \mathrm{Cl}^{2}$ & Range & & Geometric mean & $95 \% \mathrm{Cl}^{2}$ & Range \\
\hline Chloroquine & 64 & 361.8 & $266-492$ & $9-3373$ & 132 & 24.1 & $20-29$ & $5-729$ \\
\hline Quinine $^{1}$ & 63 & 128.5 & $93-175$ & $12-2174$ & - & - & - & - \\
\hline Mefloquine & 66 & 14.2 & $10-20$ & $2-723$ & 78 & 18.1 & $14-23$ & $2-337$ \\
\hline Artesunate $^{1}$ & 51 & 3.4 & $3-4$ & $0.3-52$ & - & - & - & - \\
\hline
\end{tabular}

${ }^{1}$ Performed only in P. falciparum cultures; ${ }^{2} \mathrm{Cl}$, confidence interval. 
recommended as $P$. falciparum second-line treatment by the Brazilian Malaria Control Programme.

Since 2007 the Brazilian Malaria Control Programme defined artemisinin-based combined therapy (ACT) as the first-line treatment for uncomplicated falciparum malaria. In this study, $11.7 \%$ of the isolates showed IC50 above the threshold of $10 \mathrm{nM}$ (11.7\%) for artesunate. This is surprising as these samples were collected in a period before the introduction of ACT in the area; therefore, no selection pressure is to be accounted for. A study performed in another area in the Amazonian region in the same period, showed no evidence of decreased sensitivity of local isolates to artesunate or artemether [28]. However, a recent study showed that $23.9 \%$ of the studied isolates from Gabon had a reduced susceptibility to dihydroartemisinin [16]. Decreased sensitivity of P. falciparum parasites to artemether in vitro in French Guiana and Senegal [29] as well as in vivo in Cambodia [30] has also been described. Given the immense value of artemisinin derivatives as alternative drugs for multidrug-resistant parasites, authorities must be alert to these data indicating a trend for emergence of isolates with decreased sensitivity to this drug.

The sensitivity of $P$. vivax parasites to anti-malarial drugs has not been widely monitored in vitro due the difficulties in cultivating $P$. vivax. Here, the in vitro resistance of $P$. vivax fresh isolates to chloroquine and mefloquine was evaluated for the first time in Brazil. Although the threshold of IC50 to define a sample as resistant to chloroquine is not well established for $P$. vivax, it has been proposed that the same threshold used for $P$. falciparum should be used for $P$. vivax [15]. In this case, a considerable frequency $(9.8 \%)$ of $P$. vivax isolates was above the adopted $100 \mathrm{nM}$ threshold of chloroquine. This frequency was not surprising, because $P$. vivax resistance to chloroquine has been reported in Brazil [31,32], as well as increasing numbers of severe $P$. vivax malaria cases $[33,34]$. Consequently, the use of DELI test can be an important tool for backing decisions of the Brazilian health authorities in relation to the treatment policy of $P$. vivax.

Mefloquine is usually an alternative treatment for $P$. vivax infections. Interestingly, the prevalence of $P$. vivax isolates with resistant profile to mefloquine was greater $(28 \%)$ than to P. falciparum parasites $(21.2 \%)$, corroborating a study performed in Thailand where $P$. vivax isolates were more resistant to mefloquine when compared with $P$. falciparum isolates from the same area [35].

It should be highlighted that the in vivo outcome depends on a number of factors that cannot be evaluated in vitro, including the level of innate and acquired immunity. However, in vitro assays act as a preliminary warning system indicating a trend, since in vitro resistance may be indicative of clinical resistance.

\section{Conclusions}

The data here presented using DELI test provide important and useful information regarding the sensitivity profile of Brazilian plasmodial isolates to common anti-malarial drugs. For updating and evaluating potential shifts in the profiles of drug sensitivity, periodic such studies associated with the screening using potential molecular markers should be performed.

\section{Competing interests}

The authors declare that they have no competing interests.

\section{Authors' contributions}

LRPR carried out the DELI test, performed the statistical analysis and drafted the manuscript; CTDR and LJMC conceived the study, participated in its design and coordination and helped to draft the manuscript; MJS and CBJ carried out the DELI test; YFC, MGA, PD, PB and MFFC helped in the design of the study and reviewed the manuscript. All authors have and approved the final manuscript.

\section{Acknowledgements}

We are grateful to all patients that agreed to participate in this study for their cooperation and generous donation of blood, which made this study possible. We are deeply grateful to the late Dr Michael Makler for kindly providing the 17EA and 11D monoclonal antibodies. This work was supported by Fundação de Amparo à Pesquisa do Estado do Rio de Janeiro (FAPERJ, Brazil), Conselho Nacional de Desenvolvimento Científico e Tecnológico (CNPq, Brazil), Instituto Oswaldo Cruz (FIOCRUZ, Brazil), Petróleo Brasileiro SA (Petrobrás) and the Sanofi-Aventis Access to Medicines Impact Malaria Program. CTDR and MFFC are recipients of a Research Productivity Fellowship from CNPq and receive a grant from FAPERJ as "Cientistas do Nosso Estado".

\section{Author details}

'Laboratório de Pesquisas em Malária, Instituto Oswaldo Cruz, Fiocruz, Avenida Brasil 4365, Manguinhos, Rio de Janeiro, RJ, Brazil CEP: 21040-900. ${ }^{2}$ Centro de Pesquisa, Diagnóstico e Treinamento em Malária (CPD-Mal), Fiocruz, Reference Center for Malaria in the Extra-Amazonian Region for the Secretary for Health Surveillance from the Ministry of Health, Rio de Janeiro, RJ, Brazil. ${ }^{3}$ Laboratório de Malária, Fundação de Medicina Tropical Dr Heitor Vieira Dourado, Manaus, Amazonas, Brazil. ${ }^{4}$ Institut Pasteur, Paris, France. ${ }^{5}$ UMR 198, Institut de Recherche pour le Développement, Dakar, Sénégal, Bangladesh.

Received: 23 May 2013 Accepted: 31 July 2013

Published: 12 August 2013

\section{References}

1. WHO: World malaria report. Geneva: World Health Organization; 2012. http://www.who.int/malaria/publications/world_malaria_report_2012/en/.

2. Oliveira-Ferreira J, Lacerda MV, Brasil P, Ladislau JL, Tauil PL, Daniel-Ribeiro CT: Malaria in Brazil: an overview. Malar J 2010, 9:115.

3. SVS: Boletim Epidemiológico da Malária. ; 2012. http://portal.saude.gov.br/ portal/arquivos/pdf/boletim_malaria_2010_2011.pdf.

4. Silva J: Terçã maligna cloroquino-resistente-uma série ameaça ao Hinterland brasileiro. Trib Medica 1961, 160:2-6.

5. Moore D, Lanier J: Observations on two Plasmodium falciparum infections with an abnormal response to chloroquine. Am J Trop Med Hyg 1961, 10:5-9.

6. Young M, Moore D: Chloroquine resistance in Plasmodium falciparum. Am J Trop Med Hyg 1961, 10:317-320.

7. Walker AJ, Lopez-Antunano FJ: Response to drugs of South American strains of Plasmodium falciparum. Trans R Soc Trop Med Hyg 1968, 62:654-667.

8. Gama BE, Lacerda MV, Daniel-Ribeiro CT, Ferreira-da-Cruz Mde F: Chemoresistance of Plasmodium falciparum and Plasmodium vivax parasites in Brazil: consequences on disease morbidity and control. Mem Inst Oswaldo Cruz 2011, 106(Suppl 1):159-166. 
9. Brasseur $P$, Agnamey $P$, Moreno A, Druilhe P: Evaluation of in vitro drug sensitivity of antimalarials for Plasmodium falciparum using a colorimetric assay (DELI-microtest). Med Trop (Mars) 2001, 61:545-547.

10. Druilhe $P$, Moreno $A$, Blanc $C$, Brasseur $P$, Jacquier $P$ : A colorimetric in vitro drug sensitivity assay for Plasmodium falciparum based on a highly sensitive double-site lactate dehydrogenase antigen-capture enzyme-linked immunosorbent assay. Am J Trop Med Hyg 2001, 64:233-241.

11. Moreno A, Brasseur P, Cuzin-Ouattara N, Blanc C, Druilhe P: Evaluation under field conditions of the colorimetric DELI-microtest for the assessment of Plasmodium falciparum drug resistance. Trans $R$ Soc Trop Med Hyg 2001, 95:100-103.

12. Brockman A, Singlam S, Phiaphun L, Looareesuwan S, White NJ, Nosten F: Field evaluation of a novel colorimetric method double-site enzymelinked lactate dehydrogenase immunodetection assay to determine drug susceptibilities of Plasmodium falciparum clinical isolates from northwestern Thailand. Antimicrob Agents Chemother 2004, 48:1426-1429.

13. Kaddouri H, Nakache S, Houzé S, Mentré F, Le Bras J: Assessment of the drug susceptibility of Plasmodium falciparum clinical isolates from africa by using a Plasmodium lactate dehydrogenase immunodetection assay and an inhibitory maximum effect model for precise measurement of the 50-percent inhibitory concentration. Antimicrob Agents Chemother 2006, 50:3343-3349.

14. Chotivanich K, Silamut K, Udomsangpetch R, Stepnewski K, Pukrittayakamee S, Looareesuwan S, White NJ: Ex-vivo short-term culture and developmental assessment of Plasmodium vivax. Trans R Soc Trop Med Hyg 2001, 95:677-680.

15. Druilhe P, Brasseur P, Blanc C, Makler M: Improved assessment of Plasmodium vivax response to antimalarial drugs by a colorimetric double-site plasmodium lactate dehydrogenase antigen capture enzyme-linked immunosorbent assay. Antimicrob Agents Chemother 2007, 51:2112-2116.

16. Zatra R, Lekana-douki JB, Lekoulou F, Bisvigou U, Ngoungou EB, Ndouo FS: In vitro antimalarial susceptibility and molecular markers of drug resistance in Franceville, Gabon. BMC Infect Dis 2012, 12:307.

17. Menezes CM, Kirchgatter K, Di Santi SM, Paula GA, Ferreira El: In vitro evaluation of quinidine sensitivity in Brazilian Plasmodium falciparum isolates: comparative analysis to quinine and chloroquine. Rev Inst Med Trop Sao Paulo 2001, 43:221-226.

18. Gama BE, De Oliveira NK, Zalis MG, De Souza JM, Santos F, Daniel-Ribeiro $C T$, Ferreira-da-Cruz M, De F: Chloroquine and sulphadoxinepyrimethamine sensitivity of Plasmodium falciparum parasites in a Brazilian endemic area. Malar J 2009, 8:156.

19. Alecrim MGC: Estudo da resistência do P. falciparum às drogas antimaláricas in vivo e in vitro na Amazônia, MSc thesis. Brasilia: Universidade de Brasília; 1981.

20. Couto AA, Calvosa VS, Santos MA, de Souza JM: The evolution over time of the in-vitro resistance of Plasmodium falciparum to antimalarial drugs in 2 areas of the Brazilian Amazonia with distinct socioeconomic and geographic characteristics. Rev Soc Bras Med Trop 1995, 28:357-365.

21. Cerutti Junior C, Marques C, Alencar FE, Durlacher RR, Alween A, Segurado AA, Pang LW, Zalis MG: Antimalarial drug susceptibility testing of Plasmodium falciparum in Brazil using a radioisotope method. Mem Inst Oswaldo Cruz 1999, 94:803-809.

22. Noronha E, Alecrim MG, Romero GA, Macêdo V: RIII mefloquine resistance in children with falciparum malaria in Manaus, AM, Brazil. Rev Soc Bras Med Trop 2000, 33:201-205

23. Calvosa VS, Adagu IS, Póvoa MM: Plasmodium falciparum: emerging mefloquine resistance in vitro in Para State, North Brazil. Trans R Soc Trop Med Hyg 2001, 95:330-331.

24. Boulos M, Di Santi SM, Barata LCB, Segurado AAC, Dutra AP, Neves VLF: Some aspects of treatment, prophylaxis and chemoresistance of Plasmodium falciparum malaria. Mem Inst Oswaldo Cruz 1986, 81(Suppl. II):255-257.

25. Souza JM, Sheth UK, de Oliveira RM, Roulet H, de Souza SD: An open, randomized, phase III clinical trial of mefloquine and of quinine plus sulfadoxine-pyrimethamine in the treatment of symptomatic falciparum malaria in Brazil. Bull World Health Organ 1985, 63:603-609.

26. Souza JM: Mefloquine clinical trials-therapeutical experience with mefloquine alone and combination (MSP) in Brazilian male subjects with falciparum malaria. Mem Inst Oswaldo Cruz 1986, 81(Suppl. II):259-268.

27. Zalis MG, Pang L, Silveira MS, Milhous WK, Wirth DF: Characterization of Plasmodium falciparum isolated from the Amazon region of Brazil: evidence for quinine resistance. Am J Trop Med Hyg 1998, 58:630-663.
28. Ferreira ID, Martinelli A, Rodrigues LA, Do Carmo EL, Do Rosário VE, Póvoa MM, Cravo P: Plasmodium falciparum from Pará state (Brazil) shows satisfactory in vitro response to artemisinin derivatives and absence of the S769N mutation in the SERCA-type PfATPase6. Trop Med Int Health 2008, 3:199-207.

29. Jambou R, Legrand E, Niang M, Khim N, Lim P, Volney B, Ekala MT, Bouchier C, Esterre P, Fandeur T, Mercereau-Puijalon O: Resistance of Plasmodium falciparum field isolates to in-vitro artemether and point mutations of the SERCA-type PfATPase6. Lancet 2005, 366:1960-1963.

30. Dondorp AM, Nosten F, Yi P, Das D, Phyo AP, Tarning J, Lwin KM, Ariey F, Hanpithakpong W, Lee SJ, Ringwald P, Silamut K, Imwong M, Chotivanich K, Lim P, Herdman T, An SS, Yeung S, Singhasivanon P, Day NP, Lindegardh N, Socheat D, White NJ: Artemisinin resistance in Plasmodium falciparum malaria. N Engl J Med 2009, 361:455-467.

31. Alecrim $M$, Alecrim $W$, Macedo V: Plasmodium vivax resistance to chloroquine (R2) and mefloquine (R3) in Brazilian Amazon region. Rev Soc Bras Med Trop 1999, 32:67-68.

32. Santana Filho FS, Arcanjo AR, Chehuan YM, Costa MR, Martinez-Espinosa FE, Vieira JL, Barbosa MG, Alecrim WD, Alecrim MG: Chloroquine-resistant Plasmodium vivax, Brazilian Amazon. Emerg Infect Dis 2007, 13:1125-1126.

33. Cabral PHO AS, Alecrim WD, Alecrim MGC, Lacerda MVG: Malaria and sickle cell anemia: report of complications and clinical management of three patients in a highly endemic area for Plasmodium vivax malaria in the Brazilian Amazon. Case Rep Clin Pract Rev 2006, 7:220-223.

34. Lacerda MV: Clinical manifestations and pathogenesis of the thrombocytopenia in malaria, PhD thesis. Brasilia: Universidade de Brasilia; 2007.

35. Chotivanich K, Udomsangpetch R, Chierakul W, Newton PN, Ruangveerayuth R, Pukrittayakamee A, Looareesuwan S, White N: In vitro efficacy of antimalarial drugs against Plasmodium vivax on the western border of Thailand. Am J Trop Med Hyg 2004, 70:395-397.

doi:10.1186/1475-2875-12-281

Cite this article as: Pratt-Riccio et al.: Use of a colorimetric (DELI) test for the evaluation of chemoresistance of Plasmodium falciparum and Plasmodium vivax to commonly used anti-plasmodial drugs in the Brazilian Amazon. Malaria Journal 2013 12:281.

\section{Submit your next manuscript to BioMed Central and take full advantage of:}

- Convenient online submission

- Thorough peer review

- No space constraints or color figure charges

- Immediate publication on acceptance

- Inclusion in PubMed, CAS, Scopus and Google Scholar

- Research which is freely available for redistribution

Submit your manuscript at www.biomedcentral.com/submit
C) Biomed Central 